\title{
Sum of squared logarithms - an inequality relating positive definite matrices and their matrix logarithm
}

\author{
Mircea Bîrsan ${ }^{1,2}$, Patrizio Neff ${ }^{*}$ and Johannes Lankeit ${ }^{1}$
}

${ }^{*}$ Correspondence:
patrizio.neff@uni-due.de
${ }^{1}$ Lehrstuhl für Nichtlineare Analysis
und Modellierung, Fakultät für
Mathematik, Universität
Duisburg-Essen, Essen, Germany
Full list of author information is
available at the end of the article

available at the end of the article

\author{
Abstract \\ Let $y_{1}, y_{2}, y_{3}, a_{1}, a_{2}, a_{3} \in(0, \infty)$ be such that $y_{1} y_{2} y_{3}=a_{1} a_{2} a_{3}$ and

$$
y_{1}+y_{2}+y_{3} \geq a_{1}+a_{2}+a_{3}, \quad y_{1} y_{2}+y_{2} y_{3}+y_{1} y_{3} \geq a_{1} a_{2}+a_{2} a_{3}+a_{1} a_{3}
$$

Then

$$
\left(\log y_{1}\right)^{2}+\left(\log y_{2}\right)^{2}+\left(\log y_{3}\right)^{2} \geq\left(\log a_{1}\right)^{2}+\left(\log a_{2}\right)^{2}+\left(\log a_{3}\right)^{2}
$$

This can also be stated in terms of real positive definite $3 \times 3$-matrices $P_{1}, P_{2}$ : If their determinants are equal, $\operatorname{det} P_{1}=\operatorname{det} P_{2}$, then

$$
\operatorname{tr} P_{1} \geq \operatorname{tr} P_{2} \quad \text { and } \quad \operatorname{tr} \operatorname{Cof} P_{1} \geq \operatorname{tr} \operatorname{Cof} P_{2} \quad \Longrightarrow \quad\left\|\log P_{1}\right\|_{F}^{2} \geq\left\|\log P_{2}\right\|_{F^{\prime}}^{2}
$$

where log is the principal matrix logarithm and $\|P\|_{F}^{2}=\sum_{i, j=1}^{3} P_{i j}^{2}$ denotes the Frobenius matrix norm. Applications in matrix analysis and nonlinear elasticity are indicated.

MSC: 26D05; 26D07

Keywords: matrix logarithm; elementary symmetric polynomials; inequality; characteristic polynomial; positive definite matrices; means

\section{Introduction}

Convexity is a powerful source for obtaining new inequalities; see, e.g., [1, 2]. In applications coming from nonlinear elasticity, we are faced, however, with variants of the squared logarithm function; see the last section. The function $(\log (x))^{2}$ is neither convex nor concave. Nevertheless, the sum of squared logarithms inequality holds. We will proceed as follows: In the first section, we will give several equivalent formulations of the inequality, for example, in terms of the coefficients of the characteristic polynomial (Theorem 1), in terms of elementary symmetric polynomials (Theorem 3), in terms of means (Theorem 5 ) or in terms of the Frobenius matrix norm (Theorem 7). A proof of the inequality will be given in Section 2, and some counterexamples for slightly changed variants of the inequality are discussed in Section 3. In the last section, an application of the sum of squared loga- 
rithms inequality in matrix analysis and in the mathematical theory of nonlinear elasticity is indicated.

\section{Formulations of the problem}

All theorems in this section are equivalent.

Theorem 1 For $n=2$ or $n=3$ let $P_{1}, P_{2} \in \mathbb{R}^{n \times n}$ be positive definite real matrices. Let the coefficients of the characteristic polynomials of $P_{1}$ and $P_{2}$ satisfy

$$
\operatorname{tr} P_{1} \geq \operatorname{tr} P_{2} \quad \text { and } \quad \operatorname{tr} \operatorname{Cof} P_{1} \geq \operatorname{tr} \operatorname{Cof} P_{2} \quad \text { and } \quad \operatorname{det} P_{1}=\operatorname{det} P_{2} .
$$

Then

$$
\left\|\log P_{1}\right\|_{F}^{2} \geq\left\|\log P_{2}\right\|_{F}^{2}
$$

For $n=3$, we will now give equivalent formulations of this statement. The case $n=2$ can be treated analogously. For its proof, see Remark 15. By orthogonal diagonalization of $P_{1}$ and $P_{2}$, the inequalities can be rewritten in terms of the eigenvalues $y_{1}, y_{2}, y_{3}$ and $a_{1}, a_{2}$, $a_{3}$, respectively.

Theorem 2 Let the real numbers $a_{1}, a_{2}, a_{3}>0$ and $y_{1}, y_{2}, y_{3}>0$ be such that

$$
\begin{aligned}
& y_{1}+y_{2}+y_{3} \geq a_{1}+a_{2}+a_{3}, \\
& y_{1} y_{2}+y_{2} y_{3}+y_{1} y_{3} \geq a_{1} a_{2}+a_{2} a_{3}+a_{1} a_{3}, \\
& y_{1} y_{2} y_{3}=a_{1} a_{2} a_{3} .
\end{aligned}
$$

Then

$$
\left(\log y_{1}\right)^{2}+\left(\log y_{2}\right)^{2}+\left(\log y_{3}\right)^{2} \geq\left(\log a_{1}\right)^{2}+\left(\log a_{2}\right)^{2}+\left(\log a_{3}\right)^{2} .
$$

The elementary symmetric polynomials, see, e.g., [3, p.178]

$$
\begin{aligned}
& e_{0}\left(y_{1}, y_{2}, y_{3}\right)=1, \\
& e_{1}\left(y_{1}, y_{2}, y_{3}\right)=y_{1}+y_{2}+y_{3}, \\
& e_{2}\left(y_{1}, y_{2}, y_{3}\right)=y_{1} y_{2}+y_{1} y_{3}+y_{2} y_{3}, \\
& e_{3}\left(y_{1}, y_{2}, y_{3}\right)=y_{1} y_{2} y_{3}
\end{aligned}
$$

are known to have the Schur-concavity property (i.e., $-e_{k}$ is Schur-convex) [1, 4]; see (16). It is possible to express the problem in terms of these elementary symmetric polynomials as follows.

Theorem 3 Let $a_{1}, a_{2}, a_{3}>0$ and $y_{1}, y_{2}, y_{3}>0$ satisfy

$$
\begin{aligned}
& e_{1}\left(y_{1}, y_{2}, y_{3}\right) \geq e_{1}\left(a_{1}, a_{2}, a_{3}\right), \quad e_{2}\left(y_{1}, y_{2}, y_{3}\right) \geq e_{2}\left(a_{1}, a_{2}, a_{3}\right), \\
& e_{3}\left(y_{1}, y_{2}, y_{3}\right)=e_{3}\left(a_{1}, a_{2}, a_{3}\right) .
\end{aligned}
$$


Then

$$
e_{1}\left(\left(\log y_{1}\right)^{2},\left(\log y_{2}\right)^{2},\left(\log y_{3}\right)^{2}\right) \geq e_{1}\left(\left(\log a_{1}\right)^{2},\left(\log a_{2}\right)^{2},\left(\log a_{3}\right)^{2}\right) .
$$

Because $y_{1} y_{2} y_{3}=a_{1} a_{2} a_{3}>0$, we have

$$
y_{1} y_{2}+y_{2} y_{3}+y_{1} y_{3} \geq a_{1} a_{2}+a_{2} a_{3}+a_{1} a_{3} \Leftrightarrow \frac{1}{y_{1}}+\frac{1}{y_{2}}+\frac{1}{y_{3}} \geq \frac{1}{a_{1}}+\frac{1}{a_{2}}+\frac{1}{a_{3}} .
$$

Thus, we obtain the following theorem.

Theorem 4 Let the real numbers $a_{1}, a_{2}, a_{3}>0$ and $y_{1}, y_{2}, y_{3}>0$ be such that

$$
\begin{aligned}
& y_{1}+y_{2}+y_{3} \geq a_{1}+a_{2}+a_{3}, \\
& \frac{1}{y_{1}}+\frac{1}{y_{2}}+\frac{1}{y_{3}} \geq \frac{1}{a_{1}}+\frac{1}{a_{2}}+\frac{1}{a_{3}}, \\
& y_{1} y_{2} y_{3}=a_{1} a_{2} a_{3} .
\end{aligned}
$$

Then

$$
\left(\log y_{1}\right)^{2}+\left(\log y_{2}\right)^{2}+\left(\log y_{3}\right)^{2} \geq\left(\log a_{1}\right)^{2}+\left(\log a_{2}\right)^{2}+\left(\log a_{3}\right)^{2} .
$$

The conditions (3) are also simple expressions in terms of arithmetic, harmonic and geometric and quadratic mean

$$
\begin{aligned}
& A\left(y_{1}, y_{2}, y_{3}\right)=\frac{y_{1}+y_{2}+y_{3}}{3}, \quad H\left(y_{1}, y_{2}, y_{3}\right)=\frac{3}{\frac{1}{y_{1}}+\frac{1}{y_{2}}+\frac{1}{y_{3}}}, \\
& G\left(y_{1}, y_{2}, y_{3}\right)=\sqrt[3]{y_{1} y_{2} y_{3}}, \quad Q\left(y_{1}, y_{2}, y_{3}\right)=\sqrt{\frac{1}{3}\left(y_{1}^{2}+y_{2}^{2}+y_{3}^{2}\right)} .
\end{aligned}
$$

Theorem 5 Let $a_{1}, a_{2}, a_{3}>0$ and $y_{1}, y_{2}, y_{3}>0$. Then $A\left(y_{1}, y_{2}, y_{3}\right) \geq A\left(a_{1}, a_{2}, a_{3}\right), H\left(a_{1}, a_{2}\right.$, $\left.a_{3}\right) \geq H\left(y_{1}, y_{2}, y_{3}\right)$ ('reverse!') and $G\left(y_{1}, y_{2}, y_{3}\right)=G\left(a_{1}, a_{2}, a_{3}\right)$ imply

$$
Q\left(\log y_{1}, \log y_{2}, \log y_{3}\right) \geq Q\left(\log a_{1}, \log a_{2}, \log a_{3}\right) .
$$

We denote by

$$
a_{i}=: d_{i}^{2}, \quad y_{i}=: x_{i}^{2}
$$

and arrive at

Theorem 6 Let the real numbers $d_{i}$ and $x_{i}$ be such that $d_{1}, d_{2}, d_{3}>0, x_{1}, x_{2}, x_{3}>0$ and

$$
\begin{aligned}
& x_{1}^{2}+x_{2}^{2}+x_{3}^{2} \geq d_{1}^{2}+d_{2}^{2}+d_{3}^{2}, \\
& x_{1}^{2} x_{2}^{2}+x_{2}^{2} x_{3}^{2}+x_{1}^{2} x_{3}^{2} \geq d_{1}^{2} d_{2}^{2}+d_{2}^{2} d_{3}^{2}+d_{1}^{2} d_{3}^{2}, \\
& x_{1} x_{2} x_{3}=d_{1} d_{2} d_{3} .
\end{aligned}
$$


Then

$$
\left(\log x_{1}\right)^{2}+\left(\log x_{2}\right)^{2}+\left(\log x_{3}\right)^{2} \geq\left(\log d_{1}\right)^{2}+\left(\log d_{2}\right)^{2}+\left(\log d_{3}\right)^{2} .
$$

If we again view $x_{i}$ and $d_{i}$ as eigenvalues of positive definite matrices, an equivalent formulation of the problem can be given in terms of their Frobenius matrix norms:

Theorem 7 For $n \in\{2,3\}$, let $P_{1}, P_{2} \in \mathbb{R}^{n \times n}$ be positive definite real matrices. Let

$$
\left\|P_{1}\right\|_{F}^{2} \geq\left\|P_{2}\right\|_{F}^{2} \quad \text { and } \quad\left\|P_{1}^{-1}\right\|_{F}^{2} \geq\left\|P_{2}^{-1}\right\|_{F}^{2} \quad \text { and } \quad \operatorname{det} P_{1}=\operatorname{det} P_{2} \text {. }
$$

Then

$$
\left\|\log P_{1}\right\|_{F}^{2} \geq\left\|\log P_{2}\right\|_{F}^{2}
$$

Let us reconsider the formulation from Theorem 5 . If we denote

$$
c_{i}:=\log a_{i}, \quad z_{i}:=\log y_{i},
$$

from $H\left(a_{1}, a_{2}, a_{3}\right) \geq H\left(y_{1}, y_{2}, y_{3}\right)$, we obtain

$$
e^{-z_{1}}+e^{-z_{2}}+e^{-z_{3}} \geq e^{-c_{1}}+e^{-c_{2}}+e^{-c_{3}} .
$$

Theorem 8 Let the real numbers $c_{1}, c_{2}, c_{3}$ and $z_{1}, z_{2}, z_{3}$ be such that

$$
\begin{aligned}
& e^{z_{1}}+e^{z_{2}}+e^{z_{3}} \geq e^{c_{1}}+e^{c_{2}}+e^{c_{3}}, \\
& e^{-z_{1}}+e^{-z_{2}}+e^{-z_{3}} \geq e^{-c_{1}}+e^{-c_{2}}+e^{-c_{3}}, \\
& z_{1}+z_{2}+z_{3}=c_{1}+c_{2}+c_{3} .
\end{aligned}
$$

Then

$$
z_{1}^{2}+z_{2}^{2}+z_{3}^{2} \geq c_{1}^{2}+c_{2}^{2}+c_{3}^{2} .
$$

In order to prove Theorem 8 , one can assume without loss of generality that

$$
z_{1}+z_{2}+z_{3}=c_{1}+c_{2}+c_{3}=0
$$

Thus, we have the equivalent formulation

Theorem 9 Let the real numbers $\bar{c}_{1}, \bar{c}_{2}, \bar{c}_{3}$ and $\bar{z}_{1}, \bar{z}_{2}, \bar{z}_{3}$ be such that

$$
\begin{aligned}
& e^{\bar{z}_{1}}+e^{\bar{z}_{2}}+e^{\bar{z}_{3}} \geq e^{\bar{c}_{1}}+e^{\bar{c}_{2}}+e^{\bar{c}_{3}}, \\
& e^{-\bar{z}_{1}}+e^{-\bar{z}_{2}}+e^{-\bar{z}_{3}} \geq e^{-\bar{c}_{1}}+e^{-\bar{c}_{2}}+e^{-\bar{c}_{3}}, \\
& \bar{z}_{1}+\bar{z}_{2}+\bar{z}_{3}=\bar{c}_{1}+\bar{c}_{2}+\bar{c}_{3}=0 .
\end{aligned}
$$


Then

$$
\bar{z}_{1}^{2}+\bar{z}_{2}^{2}+\bar{z}_{3}^{2} \geq \bar{c}_{1}^{2}+\bar{c}_{2}^{2}+\bar{c}_{3}^{2}
$$

Let us prove that Theorem 8 can be reformulated as Theorem 9. Indeed, let us assume that Theorem 9 is valid and show that the statement of Theorem 8 also holds true. We denote by $s$ the sum $s=z_{1}+z_{2}+z_{3}=c_{1}+c_{2}+c_{3}$ and we designate

$$
\bar{z}_{i}=z_{i}-\frac{s}{3}, \quad \bar{c}_{i}=c_{i}-\frac{s}{3} \quad(i=1,2,3) .
$$

Then the real numbers $\bar{z}_{i}$ and $\bar{c}_{i}$ satisfy the hypotheses of Theorem 9 and we obtain $\bar{z}_{1}^{2}+$ $\bar{z}_{2}^{2}+\bar{z}_{3}^{2} \geq \bar{c}_{1}^{2}+\bar{c}_{2}^{2}+\bar{c}_{3}^{2}$. This inequality is equivalent to

$$
\sum_{i=1}^{3}\left(z_{i}-\frac{s}{3}\right)^{2} \geq \sum_{i=1}^{3}\left(c_{i}-\frac{s}{3}\right)^{2}
$$

which, by virtue of the condition $(7)_{3}$, reduces to

$$
z_{1}^{2}+z_{2}^{2}+z_{3}^{2} \geq c_{1}^{2}+c_{2}^{2}+c_{3}^{2}
$$

Thus, Theorem 8 is also valid.

By virtue of the logical equivalence

$$
(A \wedge B \quad \Rightarrow \quad C) \quad \Leftrightarrow \quad(\neg C \quad \Rightarrow \quad \neg A \vee \neg B)
$$

for any statements $A, B, C$, we can formulate the inequality (11) (i.e., Theorem 9) in the following equivalent manner.

Theorem 10 Let the real numbers $c_{1}, c_{2}, c_{3}$ and $z_{1}, z_{2}, z_{3}$ be such that

$$
z_{1}+z_{2}+z_{3}=c_{1}+c_{2}+c_{3}=0 \text { and } z_{1}^{2}+z_{2}^{2}+z_{3}^{2}<c_{1}^{2}+c_{2}^{2}+c_{3}^{2}
$$

Then one of the following inequalities holds:

$$
\begin{aligned}
& e^{z_{1}}+e^{z_{2}}+e^{z_{3}}<e^{c_{1}}+e^{c_{2}}+e^{c_{3}} \quad \text { or } \\
& e^{-z_{1}}+e^{-z_{2}}+e^{-z_{3}}<e^{-c_{1}}+e^{-c_{2}}+e^{-c_{3}} .
\end{aligned}
$$

We use the statement of Theorem 10 for the proof.

Before continuing, let us show that our new inequality is not a consequence of majorization and Karamata's inequality [5]. Consider $z=\left(z_{1}, \ldots, z_{n}\right) \in \mathbb{R}_{+}^{n}$ and $c=\left(c_{1}, \ldots, c_{n}\right) \in \mathbb{R}_{+}^{n}$ arranged already in decreasing order $z_{1} \geq z_{2} \geq \cdots \geq z_{n}$ and $c_{1} \geq c_{2} \geq \cdots \geq c_{n}$. If

$$
\sum_{i=1}^{k} z_{i} \geq \sum_{i=1}^{k} c_{i} \quad(1 \leq k \leq n-1), \quad \sum_{i=1}^{n} z_{i}=\sum_{i=1}^{n} c_{i}
$$


we say that $z$ majorizes $c$, denoted by $z \succ c$. The following result is well known [6, p.89], $[4,5]$. If $f: \mathbb{R} \rightarrow \mathbb{R}$ is convex, then

$$
z \succ c \Rightarrow \sum_{i=1}^{n} f\left(z_{i}\right) \geq \sum_{i=1}^{n} f\left(c_{i}\right)
$$

A function $g: \mathbb{R}^{n} \mapsto \mathbb{R}$ which satisfies

$$
z \succ c \Rightarrow g\left(z_{1}, \ldots, z_{n}\right) \geq g\left(c_{1}, \ldots, c_{n}\right)
$$

is called Schur-convex. In Theorem 8, the convex function to be considered would be $f(t)=t^{2}$. Do conditions (7) (upon rearrangement of $z, c \in \mathbb{R}_{+}^{3}$ if necessary) yield already majorization $z \succ c$ ? This is not the case, as we explain now. Let the real numbers $z_{1} \geq z_{2} \geq$ $z_{3}$ and $c_{1} \geq c_{2} \geq c_{3}$ be such that

$$
\begin{aligned}
& e^{z_{1}}+e^{z_{2}}+e^{z_{3}} \geq e^{c_{1}}+e^{c_{2}}+e^{c_{3}}, \\
& e^{-z_{1}}+e^{-z_{2}}+e^{-z_{3}} \geq e^{-c_{1}}+e^{-c_{2}}+e^{-c_{3}}, \\
& z_{1}+z_{2}+z_{3}=c_{1}+c_{2}+c_{3} .
\end{aligned}
$$

These conditions do not imply the majorization $z \succ c$,

$$
z_{1} \geq c_{1}, \quad z_{1}+z_{2} \geq c_{1}+c_{2}, \quad z_{1}+z_{2}+z_{3}=c_{1}+c_{2}+c_{3} .
$$

Therefore, our inequality (i.e., $z_{1}^{2}+z_{2}^{2}+z_{3}^{2} \geq c_{1}^{2}+c_{2}^{2}+c_{3}^{2}$ ) does not follow from majorization in disguise.

Indeed, let

$$
z_{1}=\frac{1}{2}+\frac{0.95}{2 \sqrt{3}}, \quad z_{2}=\frac{1}{2}+\frac{0.85}{2 \sqrt{3}}, \quad z_{3}=-1-\frac{0.9}{\sqrt{3}}
$$

and

$$
c_{1}=\frac{1}{2}+\frac{1}{2 \sqrt{3}}, \quad c_{2}=-\frac{1}{2}+\frac{1}{2 \sqrt{3}}, \quad c_{3}=-\frac{1}{\sqrt{3}} .
$$

Then we have $z_{1}>z_{2}>z_{3}$ and $c_{1}>c_{2}>c_{3}$, together with

$$
\begin{aligned}
& e^{z_{1}}+e^{z_{2}}+e^{z_{3}}=4.49497 \ldots>3.57137 \ldots=e^{c_{1}}+e^{c_{2}}+e^{c_{3}}, \\
& e^{-z_{1}}+e^{-z_{2}}+e^{-z_{3}}=5.50607 \ldots>3.47107 \ldots=e^{-c_{1}}+e^{-c_{2}}+e^{-c_{3}}, \\
& z_{1}+z_{2}+z_{3}=c_{1}+c_{2}+c_{3}=0,
\end{aligned}
$$

but the majorization inequalities (18) are not satisfied, since $z_{1}<c_{1}$.

\section{Proof of the inequality}

Of course, we may assume without loss of generality that $c_{1} \geq c_{2} \geq c_{3}$ and $z_{1} \geq z_{2} \geq z_{3}$ (and the same for $\left.a_{i}, d_{i}, x_{i}, y_{i}\right)$. 
The proof begins with the crucial lemma.

Lemma 11 Let the real numbers $a \geq b \geq c$ and $x \geq y \geq z$ be such that

$$
a+b+c=x+y+z=0, \quad a^{2}+b^{2}+c^{2}=x^{2}+y^{2}+z^{2} .
$$

Then the inequality

$$
e^{a}+e^{b}+e^{c} \leq e^{x}+e^{y}+e^{z}
$$

is satisfied if and only if the relation

$$
a \leq x
$$

holds, or equivalently, if and only if

$$
c \leq z
$$

holds.

Proof Let us denote by $r:=\sqrt{\frac{2}{3}\left(a^{2}+b^{2}+c^{2}\right)}>0$. Then, from (19), it follows

$$
\begin{aligned}
& b+c=-a, \quad b^{2}+c^{2}=\frac{3}{2} r^{2}-a^{2}, \\
& y+z=-x, \quad y^{2}+z^{2}=\frac{3}{2} r^{2}-x^{2},
\end{aligned}
$$

and we find

$$
\begin{array}{ll}
b=\frac{1}{2}\left(-a+\sqrt{3\left(r^{2}-a^{2}\right)}\right), & c=\frac{1}{2}\left(-a-\sqrt{3\left(r^{2}-a^{2}\right)}\right), \\
y=\frac{1}{2}\left(-x+\sqrt{3\left(r^{2}-x^{2}\right)}\right), & z=\frac{1}{2}\left(-x-\sqrt{3\left(r^{2}-x^{2}\right)}\right) .
\end{array}
$$

In view of (19) and $a \geq b \geq c, x \geq y \geq z$, one can show that

$$
a, x \in\left[\frac{r}{2}, r\right], \quad b, y \in\left[-\frac{r}{2}, \frac{r}{2}\right], \quad c, z \in\left[-r,-\frac{r}{2}\right] .
$$

Indeed, let us verify the relations (24). We have

$$
\begin{aligned}
\frac{r}{2} \leq a \leq r & \Leftrightarrow \frac{1}{6}\left(a^{2}+b^{2}+c^{2}\right) \leq a^{2} \leq \frac{2}{3}\left(a^{2}+b^{2}+c^{2}\right) \\
& \Leftrightarrow b^{2}+c^{2} \leq 5 a^{2} \quad \text { and } a^{2} \leq 2\left(b^{2}+c^{2}\right) \\
& \Leftrightarrow b^{2}+(a+b)^{2} \leq 5 a^{2} \quad \text { and } \quad(b+c)^{2} \leq 2\left(b^{2}+c^{2}\right) \\
& \Leftrightarrow 4 a^{2}-2 a b-2 b^{2} \geq 0 \quad \text { and } \quad b^{2}+c^{2} \geq 2 b c \\
& \Leftrightarrow 2(a-b)(2 a+b) \geq 0 \quad \text { and } \quad(b-c)^{2} \geq 0,
\end{aligned}
$$


which hold true since $a \geq b$ and $2 a+b \geq a+b+c=0$. Similarly, we have

$$
\begin{aligned}
-\frac{r}{2} \leq b \leq \frac{r}{2} & \Leftrightarrow b^{2} \leq \frac{r^{2}}{4} \quad \Leftrightarrow \quad 4 b^{2} \leq \frac{2}{3}\left(a^{2}+b^{2}+c^{2}\right) \quad \Leftrightarrow \quad 5 b^{2} \leq a^{2}+c^{2} \\
& \Leftrightarrow 5 b^{2} \leq a^{2}+(a+b)^{2} \quad \Leftrightarrow \quad 2 a^{2}+2 a b-4 b^{2} \geq 0 \\
& \Leftrightarrow 2(a-b)(a+2 b) \geq 0,
\end{aligned}
$$

which holds true since $a \geq b$ and $a+2 b \geq a+b+c=0$. Also, we have

$$
\begin{aligned}
-r \leq c \leq-\frac{r}{2} & \Leftrightarrow r^{2} \geq c^{2} \geq \frac{r^{2}}{4} \quad \Leftrightarrow \quad \frac{2}{3}\left(a^{2}+b^{2}+c^{2}\right) \geq c^{2} \geq \frac{1}{6}\left(a^{2}+b^{2}+c^{2}\right) \\
& \Leftrightarrow 2\left(a^{2}+b^{2}\right) \geq c^{2} \quad \text { and } 5 c^{2} \geq a^{2}+b^{2} \\
& \Leftrightarrow 2\left(a^{2}+b^{2}\right) \geq(a+b)^{2} \quad \text { and } 5(a+b)^{2} \geq a^{2}+b^{2} \\
& \Leftrightarrow \quad(a-b)^{2} \geq 0 \quad \text { and } \quad 4 a^{2}+10 a b+4 b^{2} \geq 0 \\
& \Leftrightarrow \quad(a-b)^{2} \geq 0 \quad \text { and } \quad 2(a+2 b)(2 a+b) \geq 0,
\end{aligned}
$$

which hold true since $a+2 b \geq a+b+c=0$ and $2 a+b \geq a+b+c=0$. One can show in the same way that $x \in\left[\frac{r}{2}, r\right], y \in\left[-\frac{r}{2}, \frac{r}{2}\right], z \in\left[-r,-\frac{r}{2}\right]$, so that (24) has been verified.

We prove now that the inequality (21) holds if and only if (22) holds. Indeed, using $(23)_{2,4}$ and (24) we get

$$
\begin{aligned}
c \leq z & \Leftrightarrow \quad-a-\sqrt{3\left(r^{2}-a^{2}\right)} \leq-x-\sqrt{3\left(r^{2}-x^{2}\right)} \\
& \Leftrightarrow \quad \frac{a}{r}+\sqrt{3\left(1-\left(\frac{a}{r}\right)^{2}\right)} \geq \frac{x}{r}+\sqrt{3\left(1-\left(\frac{x}{r}\right)^{2}\right)} \Leftrightarrow a \leq x,
\end{aligned}
$$

since the function $t \mapsto t+\sqrt{3\left(1-t^{2}\right)}$ is decreasing for $t \in\left[\frac{1}{2}, 1\right]$.

Let us prove next that the inequalities (20) and (21) are equivalent. To accomplish this, we introduce the function $f:\left[\frac{r}{2}, r\right] \rightarrow \mathbb{R}$ by

$$
f(x)=e^{x}+e^{\left(-x+\sqrt{3\left(r^{2}-x^{2}\right)}\right) / 2}+e^{\left(-x-\sqrt{3\left(r^{2}-x^{2}\right)}\right) / 2} .
$$

Taking into account (23) and $(24)_{1}$, the inequality (20) can be written equivalently as

$$
f(a) \leq f(x)
$$

which is equivalent to

$$
a \leq x,
$$

since the function $f$ defined by (25) is monotone increasing on $\left[\frac{r}{2}, r\right]$, as we show next. To this aim, we denote by

$$
\cos \varphi:=\frac{x}{r} \in\left[\frac{1}{2}, 1\right], \text { i.e. } \varphi:=\arccos \left(\frac{x}{r}\right) \in\left[0, \frac{\pi}{3}\right] .
$$


Then the function (25) can be written as

$$
\begin{aligned}
& f(x)=h(r, \varphi), \quad \text { where } h:(0, \infty) \times\left[0, \frac{\pi}{3}\right] \rightarrow \mathbb{R}, \\
& h(r, \varphi)=e^{r \cos \varphi}+e^{r \cos (\varphi+2 \pi / 3)}+e^{r \cos (\varphi-2 \pi / 3)} .
\end{aligned}
$$

We have to show that $h(r, \varphi)$ is decreasing with respect to $\varphi \in\left[0, \frac{\pi}{3}\right]$. We compute the first derivative

$$
\begin{aligned}
& \frac{\partial}{\partial \varphi} h(r, \varphi) \\
& \quad=-r\left[e^{r \cos \varphi} \sin \varphi+e^{r \cos (\varphi+2 \pi / 3)} \sin \left(\varphi+\frac{2 \pi}{3}\right)+e^{r \cos (\varphi-2 \pi / 3)} \sin \left(\varphi-\frac{2 \pi}{3}\right)\right] .
\end{aligned}
$$

The function (28) has the same sign as the function

$$
F(r, \varphi):=\frac{1}{r} e^{-r \cos \varphi} \frac{\partial}{\partial \varphi} h(r, \varphi),
$$

i.e., the function $F:(0, \infty) \times\left[0, \frac{\pi}{3}\right] \rightarrow \mathbb{R}$ given by

$$
F(r, \varphi)=-\sin \varphi-e^{-r \sqrt{3} \sin (\varphi+\pi / 3)} \sin \left(\varphi+\frac{2 \pi}{3}\right)-e^{r \sqrt{3} \sin (\varphi-\pi / 3)} \sin \left(\varphi-\frac{2 \pi}{3}\right) .
$$

In order to show that $F(r, \varphi) \leq 0$ for all $(r, \varphi) \in(0, \infty) \times\left[0, \frac{\pi}{3}\right]$, we remark that $\lim _{r \searrow 0} F(r, \varphi)=0$ for fixed $\varphi \in\left[0, \frac{\pi}{3}\right]$ and we compute

$$
\begin{aligned}
\frac{\partial}{\partial r} F(r, \varphi)= & \sqrt{3}\left[e^{-r \sqrt{3} \sin (\varphi+\pi / 3)} \sin \left(\varphi+\frac{\pi}{3}\right) \sin \left(\varphi+\frac{2 \pi}{3}\right)\right. \\
& \left.-e^{r \sqrt{3} \sin (\varphi-\pi / 3)} \sin \left(\varphi-\frac{\pi}{3}\right) \sin \left(\varphi-\frac{2 \pi}{3}\right)\right] \\
= & \sqrt{3}\left[e^{-r \sqrt{3} \sin (\varphi+\pi / 3)} \frac{1}{2}\left(-\cos (2 \varphi+\pi)+\cos \frac{\pi}{3}\right)\right. \\
& \left.-e^{r \sqrt{3} \sin (\varphi-\pi / 3)} \frac{1}{2}\left(-\cos (2 \varphi-\pi)+\cos \frac{-\pi}{3}\right)\right] \\
= & \frac{\sqrt{3}}{2}\left(\cos 2 \varphi+\frac{1}{2}\right)\left[e^{-r \sqrt{3} \sin (\varphi+\pi / 3)}-e^{r \sqrt{3} \sin (\varphi-\pi / 3)}\right] \leq 0,
\end{aligned}
$$

since $\varphi \in\left[0, \frac{\pi}{3}\right]$ implies $\cos 2 \varphi \geq-\frac{1}{2}$ and $-\sin \left(\varphi+\frac{\pi}{3}\right) \leq \sin \left(\varphi-\frac{\pi}{3}\right)$.

Consequently, the function $F(r, \varphi)$ is decreasing with respect to $r$ and for any $(r, \varphi) \in$ $(0, \infty) \times\left[0, \frac{\pi}{3}\right]$ we have that

$$
F(r, \varphi) \leq \lim _{r \searrow 0} F(r, \varphi)=0
$$

From (29) and (31), it follows that $h(r, \varphi)$ is decreasing with respect to $\varphi \in\left[0, \frac{\pi}{3}\right]$. This means that $f(x)$ is increasing as a function of $x \in\left[\frac{r}{2}, r\right]$, i.e., the relation (26) is indeed equivalent to $a \leq x$ and the proof is complete. 
Consequence 12 Let the real numbers $a \geq b \geq c$ and $x \geq y \geq z$ be such that

$$
a+b+c=x+y+z=0, \quad a^{2}+b^{2}+c^{2}=x^{2}+y^{2}+z^{2} .
$$

Then one of the following inequalities holds:

$$
e^{a}+e^{b}+e^{c} \leq e^{x}+e^{y}+e^{z}
$$

or

$$
e^{-a}+e^{-b}+e^{-c} \leq e^{-x}+e^{-y}+e^{-z} .
$$

The inequalities (32) and (33) are satisfied simultaneously if and only if $a=x, b=y$ and $c=z$.

Proof According to Lemma 11, the inequality (32) is equivalent to

$$
a \leq x,
$$

while the inequality (33) is equivalent to

$$
-a \leq-x .
$$

Since one of the relations (34) and (35) must hold, we have proved that one of the inequalities (32) and (33) is satisfied. They are simultaneously satisfied if and only if both (34) and (35) hold true, i.e., $a=x$ (and consequently $b=y, c=z$ ).

Consequence 13 Let the real numbers $a \geq b \geq c$ and $x \geq y \geq z$ be such that

$$
\begin{aligned}
& a+b+c=x+y+z=0, \quad a^{2}+b^{2}+c^{2}=x^{2}+y^{2}+z^{2} \\
& \text { and } e^{a}+e^{b}+e^{c}=e^{x}+e^{y}+e^{z} .
\end{aligned}
$$

Then we have $a=x, b=y$ and $c=z$.

Proof Since by hypothesis $e^{a}+e^{b}+e^{c} \leq e^{x}+e^{y}+e^{z}$ holds, we can apply Lemma 11 to deduce $a \leq x$ and $c \leq z$.

On the other hand, by virtue of the inverse inequality $e^{x}+e^{y}+e^{z} \leq e^{a}+e^{b}+e^{c}$ and Lemma 11, we obtain $x \leq a$ and $z \leq c$. In conclusion, we get $a=x, c=z$ and $b=y$.

Proof of Theorem 10 In order to prove (13), we define the real numbers

$$
t_{i}=k z_{i} \quad(i=1,2,3), \quad \text { where } k=\sqrt{\frac{c_{1}^{2}+c_{2}^{2}+c_{3}^{2}}{z_{1}^{2}+z_{2}^{2}+z_{3}^{2}}}>1 .
$$

Then we have

$$
t_{1}+t_{2}+t_{3}=c_{1}+c_{2}+c_{3}=0 \text { and } t_{1}^{2}+t_{2}^{2}+t_{3}^{2}=c_{1}^{2}+c_{2}^{2}+c_{3}^{2} .
$$


If we apply the Consequence 12 for the numbers $c_{1} \geq c_{2} \geq c_{3}$ and $t_{1} \geq t_{2} \geq t_{3}$, then we obtain that

$$
\begin{aligned}
& e^{t_{1}}+e^{t_{2}}+e^{t_{3}} \leq e^{c_{1}}+e^{c_{2}}+e^{c_{3}} \quad \text { or } \\
& e^{-t_{1}}+e^{-t_{2}}+e^{-t_{3}} \leq e^{-c_{1}}+e^{-c_{2}}+e^{-c_{3}} .
\end{aligned}
$$

In what follows, let us show that

$$
e^{z_{1}}+e^{z_{2}}+e^{z_{3}}<e^{t_{1}}+e^{t_{2}}+e^{t_{3}} .
$$

Using the notations $\rho:=\sqrt{\frac{2}{3}\left(z_{1}^{2}+z_{2}^{2}+z_{3}^{2}\right)}$ and

$$
\cos \zeta:=\frac{z_{1}}{\rho} \in\left[\frac{1}{2}, 1\right], \quad \text { i.e. } \zeta:=\arccos \left(\frac{z_{1}}{\rho}\right) \in\left[0, \frac{\pi}{3}\right]
$$

we have $k \rho:=\sqrt{\frac{2}{3}\left(t_{1}^{2}+t_{2}^{2}+t_{3}^{2}\right)}$ and $\cos \zeta=\frac{t_{1}}{k \rho}$. With the help of the function $h$ defined in (27), we can write the inequality (39) in the form

$$
\begin{aligned}
& e^{\rho \cos \zeta}+e^{\rho \cos (\zeta+2 \pi / 3)}+e^{\rho \cos (\zeta-2 \pi / 3)}<e^{k \rho \cos \zeta}+e^{k \rho \cos (\zeta+2 \pi / 3)}+e^{k \rho \cos (\zeta-2 \pi / 3)}, \quad \text { or } \\
& h(\rho, \zeta)<h(k \rho, \zeta), \quad \forall(\rho, \zeta) \in(0, \infty) \times\left[0, \frac{\pi}{3}\right], \quad k>1 .
\end{aligned}
$$

The relation (40) asserts that the function $h$ defined in (27) is increasing with respect to the first variable $r \in(0, \infty)$. To show this, we compute the derivative

$$
\frac{\partial}{\partial r} h(r, \varphi)=e^{r \cos \varphi} \cos \varphi+e^{r \cos (\varphi+2 \pi / 3)} \cos \left(\varphi+\frac{2 \pi}{3}\right)+e^{r \cos (\varphi-2 \pi / 3)} \cos \left(\varphi-\frac{2 \pi}{3}\right) .
$$

By virtue of the Chebyshev's sum inequality, we deduce from (41) that

$$
\frac{\partial}{\partial r} h(r, \varphi)>0 \text {. }
$$

Indeed, the Chebyshev's sum inequality $[6,2.17]$ asserts that: if $a_{1} \geq a_{2} \geq \cdots \geq a_{n}$ and $b_{1} \geq b_{2} \geq \cdots \geq b_{n}$ then

$$
n \sum_{k=1}^{n} a_{k} b_{k} \geq\left(\sum_{k=1}^{n} a_{k}\right)\left(\sum_{k=1}^{n} b_{k}\right) .
$$

In our case, we derive the following result: for any real numbers $x, y, z$ such that $x+y+z=0$, the inequality

$$
x e^{x}+y e^{y}+z e^{z} \geq \frac{1}{3}(x+y+z)\left(e^{x}+e^{y}+e^{z}\right)=0,
$$

holds true, with equality if and only if $x=y=z=0$.

Applying the result (43) to the function (41), we deduce the relation (42). This means that $h(r, \varphi)$ is an increasing function of $r$, i.e. the inequality (40) holds, and hence, we have proved (39). 
One can show analogously that the inequality

$$
e^{-z_{1}}+e^{-z_{2}}+e^{-z_{3}}<e^{-t_{1}}+e^{-t_{2}}+e^{-t_{3}}
$$

is also valid. From (38), (39) and (44), it follows that the assertion (13) holds true. Thus, the proof of Theorem 10 is complete.

Since the statements of the Theorems 8 and 10 are equivalent, we have proved also the inequality (8).

Remark 14 The inequality (8) becomes an equality if and only if $z_{i}=c_{i}, i=1,2,3$.

Proof Indeed, assume that $z_{1}^{2}+z_{2}^{2}+z_{3}^{2}=c_{1}^{2}+c_{2}^{2}+c_{3}^{2}$. Then we can apply the Consequence 12 and we deduce that

$$
e^{z_{1}}+e^{z_{2}}+e^{z_{3}} \leq e^{c_{1}}+e^{c_{2}}+e^{c_{3}} \text { or } \quad e^{-z_{1}}+e^{-z_{2}}+e^{-z_{3}} \leq e^{-c_{1}}+e^{-c_{2}}+e^{-c_{3}} .
$$

Taking into account (7) 1,2 in conjunction with (45), we find

$$
e^{z_{1}}+e^{z_{2}}+e^{z_{3}}=e^{c_{1}}+e^{c_{2}}+e^{c_{3}} \quad \text { or } \quad e^{-z_{1}}+e^{-z_{2}}+e^{-z_{3}}=e^{-c_{1}}+e^{-c_{2}}+e^{-c_{3}} .
$$

By virtue of (46), we can apply the Consequence 13 to derive $z_{1}=c_{1}$, and consequently $z_{2}=c_{2}, z_{3}=c_{3}$.

Let us prove the following version of the inequality (6) for two pairs of numbers $d_{1}, d_{2}$ and $x_{1}, x_{2}$ :

Remark 15 If the real numbers $d_{1} \geq d_{2}>0$ and $x_{1} \geq x_{2}>0$ are such that

$$
x_{1}^{2}+x_{2}^{2} \geq d_{1}^{2}+d_{2}^{2} \quad \text { and } \quad x_{1} x_{2}=d_{1} d_{2}=1
$$

then the inequality

$$
\left(\log x_{1}\right)^{2}+\left(\log x_{2}\right)^{2} \geq\left(\log d_{1}\right)^{2}+\left(\log d_{2}\right)^{2}
$$

holds true. Note that the additional condition

$$
\frac{1}{x_{1}^{2}}+\frac{1}{x_{2}^{2}} \geq \frac{1}{d_{1}^{2}}+\frac{1}{d_{2}^{2}}
$$

is automatically fulfilled.

Proof Since $x_{1} x_{2}=d_{1} d_{2}=1$ and $d_{1} \geq d_{2}>0, x_{1} \geq x_{2}>0$, we have $x_{1} \geq 1, d_{1} \geq 1$ and

$$
\log x_{1}=-\log x_{2} \geq 0, \quad \log d_{1}=-\log d_{2} \geq 0,
$$

so that the inequality (48) is equivalent to $\log x_{1} \geq \log d_{1}$, i.e., we have to show that $x_{1} \geq d_{1}$. 
Indeed, if we insert $x_{2}=\frac{1}{x_{1}}$ and $d_{2}=\frac{1}{d_{1}}$ into the inequality $(47)_{1}$ then we find

$$
x_{1}^{2}+\frac{1}{x_{1}^{2}} \geq d_{1}^{2}+\frac{1}{d_{1}^{2}},
$$

which means that $x_{1} \geq d_{1}$ since the function $t \mapsto t^{2}+\frac{1}{t^{2}}$ is increasing for $t \in[1, \infty)$. This completes the proof.

Alternative proof of Remark 15 Let $x_{3}=d_{3}=1$. Then (47) implies $x_{1}^{2}+x_{2}^{2}+x_{3}^{2} \geq d_{1}^{2}+d_{2}^{2}+d_{3}^{2}$ and $x_{1} x_{2} x_{3}=d_{1} d_{2} d_{3}=1$ as well as

$$
x_{1}^{2} x_{2}^{2}+x_{2}^{2} x_{3}^{2}+x_{1}^{2} x_{3}^{2}=1+x_{2}^{2}+x_{1}^{2} \geq 1+d_{2}^{2}+d_{1}^{2}=d_{1}^{2} d_{2}^{2}+d_{2}^{2} d_{3}^{2}+d_{1}^{2} d_{3}^{2}
$$

because $x_{1}^{2} x_{2}^{2}=1=d_{1}^{2} d_{2}^{2}$, and Theorem 6 provides the assertion.

\section{Some counterexamples for weakened assumptions}

Example 16 Unlike in the 2D case in Remark 15, for two triples of numbers the second condition (18) $)_{2}$ of Theorem 2 , namely $y_{1} y_{2}+y_{2} y_{3}+y_{1} y_{3} \geq a_{1} a_{2}+a_{2} a_{3}+a_{1} a_{3}$, cannot be removed. Let

$$
y_{1}=e^{6}, \quad y_{2}=1, \quad y_{3}=e^{-6}, \quad a_{1}=e^{4}, \quad a_{2}=e^{4}, \quad a_{3}=e^{-8} .
$$

Then $y_{1} y_{2} y_{3}=a_{1} a_{2} a_{3}=1$ and

$$
y_{1}+y_{2}+y_{3}>e^{6} \geq e^{2} e^{4}>3 e^{4}>a_{1}+a_{2}+a_{3},
$$

but

$$
\begin{aligned}
\left(\log y_{1}\right)^{2}+\left(\log y_{2}\right)^{2}+\left(\log y_{3}\right)^{2} & =36+0+36 \\
& <16+16+64=\left(\log a_{1}\right)^{2}+\left(\log a_{2}\right)^{2}+\left(\log a_{3}\right)^{2}
\end{aligned}
$$

Example 17 The condition $y_{1} y_{2} y_{3}=a_{1} a_{2} a_{3}$ cannot be weakened to $y_{1} y_{2} y_{3} \geq a_{1} a_{2} a_{3}$. Indeed, let $y_{2}=y_{3}=a_{1}=a_{2}=1, y_{1}=e, a_{3}=e^{-2}$. Then

$$
\begin{aligned}
& y_{1}+y_{2}+y_{3}=e+1+1 \geq 1+1+e^{-2}=a_{1}+a_{2}+a_{3}, \\
& y_{1} y_{2}+y_{1} y_{3}+y_{2} y_{3}=e+e+1 \geq 1+e^{-2}+e^{-2}=a_{1} a_{2}+a_{1} a_{3}+a_{2} a_{3}, \\
& y_{1} y_{2} y_{3}=e \geq e^{-2}=a_{1} a_{2} a_{3} .
\end{aligned}
$$

But nevertheless

$$
\left(\log y_{1}\right)^{2}+\left(\log y_{2}\right)^{2}+\left(\log y_{3}\right)^{2}=1+0+0<0+0+4=\left(\log a_{1}\right)^{2}+\left(\log a_{2}\right)^{2}+\left(\log a_{3}\right)^{2}
$$

A counterexample for the two variable case can be constructed analogously. 
Example 18 Even with an analogous condition, the inequality (4) does not hold for $n=4$ numbers (without further assumptions). Indeed, let

$$
y_{1}=e, \quad y_{2}=y_{3}=e^{7}, \quad y_{4}=e^{-15}, \quad a_{1}=a_{2}=e^{6}, \quad a_{3}=e^{7}, \quad a_{4}=e^{-19} .
$$

Then $y_{1} y_{2} y_{3} y_{4}=a_{1} a_{2} a_{3} a_{4}=1$. Also,

$$
y_{1}+y_{2}+y_{3}+y_{4}=e+e^{7}+e^{7}+e^{-15}>0+e^{7}+2 e^{6}+e^{-19}=a_{1}+a_{2}+a_{3}+a_{4} .
$$

Furthermore,

$$
y_{1} y_{2}+y_{1} y_{3}+y_{1} y_{4}+y_{2} y_{3}+y_{2} y_{4}+y_{3} y_{4}=e^{8}+e^{8}+e^{-14}+e^{14}+e^{-8}+e^{-8}
$$

and

$$
a_{1} a_{2}+a_{1} a_{3}+a_{1} a_{4}+a_{2} a_{3}+a_{2} a_{4}+a_{3} a_{4}=e^{12}+e^{13}+e^{-13}+e^{13}+e^{-13}+e^{-12} .
$$

Since $e^{2}>2 e+1$, we have $e^{14}>e^{13}+e^{13}+e^{12}$ and, therefore,

$$
y_{1} y_{2}+y_{1} y_{3}+y_{1} y_{4}+y_{2} y_{3}+y_{2} y_{4}+y_{3} y_{4} \geq a_{1} a_{2}+a_{1} a_{3}+a_{1} a_{4}+a_{2} a_{3}+a_{2} a_{4}+a_{3} a_{4} .
$$

Nevertheless, for the sum of squared logarithms, the 'reverse' inequality

$$
\begin{aligned}
\left(\log y_{1}\right)^{2}+\left(\log y_{2}\right)^{2}+\left(\log y_{3}\right)^{2}+\left(\log y_{4}\right)^{2} & =1+49+49+225=324 \\
& <482=36+36+49+361 \\
& =\left(\log a_{1}\right)^{2}+\left(\log a_{2}\right)^{2}+\left(\log a_{3}\right)^{2}+\left(\log a_{4}\right)^{2}
\end{aligned}
$$

holds true.

Example 19 The inequality (4) does not remain true either, if the function $\log (y)$ is replaced by its linearization $(y-1)$. Indeed, let $y_{1}=9, y_{2}=5, y_{3}=\frac{1}{45}, a_{1}=10, a_{2}=1, a_{3}=\frac{1}{10}$. Then

$$
y_{1}+y_{2}+y_{3}>14>11.1=a_{1}+a_{2}+a_{3}
$$

and

$$
y_{1} y_{2}+y_{1} y_{3}+y_{2} y_{3}>45 \geq 11.1=a_{1} a_{2}+a_{1} a_{3}+a_{2} a_{3} .
$$

But

$$
\begin{aligned}
\left(y_{1}-1\right)^{2}+\left(y_{2}-1\right)^{2}+\left(y_{3}-1\right)^{2} & =64+16+\left(\frac{44}{45}\right)^{2}<81<9^{2}+0+\left(\frac{9}{10}\right)^{2} \\
& =\left(a_{1}-1\right)^{2}+\left(a_{2}-1\right)^{2}+\left(a_{3}-1\right)^{2} .
\end{aligned}
$$




\section{Conjecture for arbitrary $n$}

The structure of the inequality in dimensions $n=2$ and $n=3$ and extensive numerical sampling strongly suggest that the inequality holds for all $n \in \mathbb{N}$ if the $n$ corresponding conditions are satisfied. More precisely, in terms of the elementary symmetric polynomials, we expect the following:

Conjecture 20 Let $n \in \mathbb{N}$ and $y_{i}, a_{i}>0$ for $i=1, \ldots, n$. If for all $i=1, \ldots, n-1$ we have

$$
e_{i}\left(y_{1}, \ldots, y_{n}\right) \geq e_{i}\left(a_{1}, \ldots, a_{n}\right) \text { and } e_{n}\left(y_{1}, \ldots, y_{n}\right)=e_{n}\left(a_{1}, \ldots, a_{n}\right) \text {, }
$$

then

$$
\sum_{i=1}^{n}\left(\log y_{i}\right)^{2} \geq \sum_{i=1}^{n}\left(\log a_{i}\right)^{2}
$$

\section{Applications}

The investigation in this paper has been motivated by some recent applications. The new sum of squared logarithms inequality is one of the fundamental tools in deducing a novel optimality result in matrix analysis and the conditions in the form (3) had been deduced in the course of that work. Optimality in the matrix problem suggested the sum of squared logarithms inequality. Indeed, based on the present result in [7], it has been shown that for all invertible $Z \in \mathbb{C}^{3 \times 3}$ and for any definition of the matrix logarithm as possibly multivalued solution $X \in \mathbb{C}^{3 \times 3}$ of $\exp X=Z$ it holds

$$
\begin{aligned}
& \min _{Q^{*} Q=I}\left\|\log Q^{*} Z\right\|_{F}^{2}=\left\|\log U_{p}^{*} Z\right\|_{F}^{2}=\|\log H\|_{F}^{2}, \\
& \min _{Q * Q=I}\left\|\operatorname{sym} \log Q^{*} Z\right\|_{F}^{2}=\left\|\operatorname{sym} \log U_{p}^{*} Z\right\|_{F}^{2}=\|\log H\|_{F}^{2},
\end{aligned}
$$

where $\operatorname{sym} X=\frac{1}{2}\left(X+X^{*}\right)$ is the Hermitian part of $X \in \mathbb{C}^{3 \times 3}$ and $U_{p}$ is the unitary factor in the polar decomposition of $Z$ into unitary and Hermitian positive definite matrix $H$

$$
Z=U_{p} H
$$

This result (50) generalizes the fact that for any complex logarithm and for all $z \in \mathbb{C} \backslash\{0\}$

$$
\min _{\vartheta \in(-\pi, \pi]}\left|\log _{\mathbb{C}}\left[e^{-i \vartheta} z\right]\right|^{2}=\left|\log _{\mathbb{R}}\right| z||^{2}, \quad \min _{\vartheta \in(-\pi, \pi]}\left|\mathfrak{R e} \log _{\mathbb{C}}\left[e^{-i \vartheta} z\right]\right|^{2}=\left|\log _{\mathbb{R}}\right| z||^{2}
$$

The optimality result (50) can now also be viewed as another characterization of the unitary factor in the polar decomposition. In addition, in a forthcoming contribution [8], we use (50) to calculate the geodesic distance of the isochoric part of the deformation gradient $\frac{F}{\operatorname{det} F^{\frac{1}{3}}} \in \mathrm{SL}(3, \mathbb{R})$ to $\mathrm{SO}(3, \mathbb{R})$ in the canonical left-invariant Riemannian metric on $\operatorname{SL}(3, \mathbb{R})$, to the effect that

$$
\operatorname{dist}_{\text {geod }}^{2}\left(\frac{F}{\operatorname{det} F^{\frac{1}{3}}}, \operatorname{SO}(3, \mathbb{R})\right)=\left\|\operatorname{dev}_{3} \log \sqrt{F^{T} F}\right\|_{F}^{2},
$$


where $\operatorname{dev}_{3} X=X-\frac{1}{3}(\operatorname{tr} X) I$ is the orthogonal projection of $X \in \mathbb{R}^{3 \times 3}$ to trace free matrices. Thereby, we provide a rigorous geometric justification for the preferred use of the Henckystrain measure $\left\|\log \sqrt{F^{T} F}\right\|_{F}^{2}$ in nonlinear elasticity and plasticity theory [9].

\section{Competing interests}

The authors declare that they have no competing interests.

Authors' contributions

All authors contributed fully to all parts of the manuscript. Notably all ideas have emerged by continuous discussions among them.

\section{Author details}

${ }^{1}$ Lehrstuhl für Nichtlineare Analysis und Modellierung, Fakultät für Mathematik, Universität Duisburg-Essen, Essen Germany. ${ }^{2}$ Department of Mathematics, University 'A.I. Cuza' of Iaşi, Iaşi, Romania.

\section{Acknowledgements}

The first author (MB) was supported by the German state grant: 'Programm des Bundes und der Länder für bessere Studienbedingungen und mehr Qualität in der Lehre'.

\section{Received: 21 January 2013 Accepted: 28 March 2013 Published: 12 April 2013}

\section{References}

1. Guan, K: Schur-convexity of the complete elementary symmetric functions. J. Inequal. Appl. 2006, Article ID 67624 (2006). doi:10.1155/JIA/2006/67624

2. Roventa, I: A note on Schur-concave functions. J. Inequal. Appl. 159, 1-9 (2012)

3. Steele, JM: The Cauchy-Schwarz Master Class: an Introduction to the Art of Mathematical Inequalities. Cambridge University Press, Cambridge (2004)

4. Khan, AR, Latif, N, Pečarić, J: Exponential convexity for majorization. J. Inequal. Appl. 105, 1-13 (2012)

5. Karamata, J: Sur une inégalité relative aux fonctions convexes. Publ. Math. Univ. Belgrad 1, 145-148 (1932)

6. Hardy, GH, Littlewood, JE, Pólya, G: Inequalities. The University Press, Cambridge (1934)

7. Neff, $P$, Nagatsukasa, $Y$, Fischle, $A$ : The unitary polar factor $Q=U_{p}$ minimizes $\left\|\log \left(Q^{*} Z\right)\right\|^{2}$ and $\left\|\operatorname{sym} \log _{*}\left(Q^{*} Z\right)\right\|^{2}$ in the spectral norm in any dimension and the Frobenius matrix norm in three dimensions (2013, submitted)

8. Neff, P, Eidel, B, Osterbrink, F, Martin, R: The isotropic Hencky strain energy $\|$ log $U \|^{2}$ measures the geodesic distance of the deformation gradient $F \in \mathrm{GL}^{+}(n)$ to $\mathrm{SO}(n)$ in the unique left-invariant Riemannian metric on $\mathrm{GL}^{+}(n)$ which is also right $O(n)$-invariant (2013, in preparation)

9. Hencky, H: Über die Form des Elastizitätsgesetzes bei ideal elastischen Stoffen. Z. Techn. Physik 9, $215-220$ (1928)

doi:10.1186/1029-242X-2013-168

Cite this article as: Bîrsan et al.: Sum of squared logarithms - an inequality relating positive definite matrices and their matrix logarithm. Journal of Inequalities and Applications 2013 2013:168.

\section{Submit your manuscript to a SpringerOpen ${ }^{\circ}$ journal and benefit from:}

- Convenient online submission

Rigorous peer review

- Immediate publication on acceptance

- Open access: articles freely available online

- High visibility within the field

- Retaining the copyright to your article 